\title{
Hydrodynamics of Cathode Films
}

\author{
Garbis H. Keulegan
}

\begin{abstract}
A hydrodynamical theory of the cathode diffusion layer based on the assumption of a uniform density reduction at the cathode surface is presented. By means of an approximate solution for the flow and the diffusion equations, the laver dimensions, the velocity distributions, and the densities are determined. Further derivations from the solutions directly applicable to results obtained by the freezing method are effected. The data of Brenner on copper and nickel baths are considered. Using the observed values of the density reductions at the cathode surface, the maximum width of the layer, the average densities for a given distance from the cathode, and the mobilities of the cations are determined and compared with the observations. The agreement between observed and computed values is regarded as fairly satisfactory.
\end{abstract}

\section{Introduction}

Neither the cognizance of the phenomenon representing the subject matter of the present paper, nor the statement of the basic causes producing it is original with us. Some 12 years ago, W. Blum, Chief of the Electrodeposition Section, National Bureau of Standards, described to us the cathode film phenomenon as a thin layer of moving material very close to the cathode surface and as being caused by the continued reduction of the densities due to electrodeposition. Since the phenomenon in question is also partly hydrodynamical in nature, he suggested that we should prepare a theoretical study with a view to determining the density distributions in cathode films.

Blum's first thought was that the film might constitute a seat of discontinuity in densities, since to the naked eye a line of demarcation between the contiguous layers of liquid near the cathode surface is distinctly visible. The line of demarcation should be due to the different indices of refractions of the liquid layers on the two sides of the line. Thus, according to these ideas the flow near the cathode surface is in one or two layers having dissimilar densities with sharp discontinuities. But, despite the appearance of the region close to the cathode surface, the opinion was ventured on our part that the exsitence of the sharp discontinuities could not be shown on the basis of purely hydrodynamical reasoning. The basis for this opinion was the belief that the phenomenon in question was essentially similar to the type of flow encountered when a heated vertical plate in the presence of cold air sets up convection currents. Since experience in this particular case does not show any discontinuities in temperatures, it might be expected that density discontinuities would not be present in the cathode plate problem. Unfortunately, due to other pressing work at that time, it was impossible to proceed with a mathematical solution of this interesting problem.

About 3 years ago, Blum repeated his request for an analytical study of the problem, since he was planning to resume the experimental investigation of cathode films. The computations that appear in this paper were completed shortly after the receipt of this request. Meanwhile two other papers on the subject, one by Agar [1] ${ }^{1}$ and the other by Wagner [2], were published. Since the present work proceeds on a mathematical basis different from these two papers and since the work was conceived with a view of interpreting the results of A. Brenner, a member of the staff of the Bureau, on cathode film densities, it is hoped that some interest in the results achieved will develop.

In the present treatment it is assumed that the reduction of densities over the vertical cathode surface is constant. On the whole the treatment is self-contained and is given in full detail. In general, the results shown are approximate and sufficient, it is believed, to evaluate average densities in a vertical plane parallel to the cathode surface. In a future publication it is proposed to investigate the problem of cathode films with the basic assumption that the density gradient at the cathode surface is constant and uniform, rather than the density.

\section{Molecular Character of Electric Conduction in Solutions}

Certain fundamental relations of electrochemical import that will be considered during the course of the analysis are based on the ideas of Larmor [3] in the matter of electrolytic conduction.

Taking an idealized situation it will be supposed that the electrolytic solution is a binary system and that ionization is complete. In each portion of the solution the number of the positive ions and the negative ions is the same and the conditions are steady, that is, independent of time. At a point of the solution let the number of ions of each kind per unit volume be $n$. Measuring positive $y$ horizontally away from the plane cathode surface toward the anode, let a section normal to $y$ be selected. Let $d N_{1}$ be the number of the cations and $d N_{2}$ the number of anions crossing the section per unit area during time $d t$. The motions of the ions are due in

Figures in brackets indicate the literature references at the end of this paper. 
part to the electrical force $d P / d y, P$ being the electrical potential, and in part to the forces producing diffusion. If $U_{1}$ be the specific mobility of the cations and $k_{1}$ the coefficient of diffusion of these ions,

$$
d N_{1}=\left(n U_{1} \frac{d P}{d y}+k_{1} \frac{d n}{d y}\right) d t
$$

and for the anions, in similar manner,

$$
d N_{2}=\left(n U_{2} \frac{d P}{d y}-k_{2} \frac{d n}{d y}\right) d t .
$$

Since there is no accumulation of electricity at a point, the relation between the electrical current intensity, I, and the motion of the ions will be

$$
\operatorname{se}\left(d N_{1}+d N_{2}\right)=I d t,
$$

where se is the charge on each ion. Applying the principle of continuity of the ionic flows,

$$
\frac{1}{2} \frac{d}{d y}\left(\frac{d N_{1}}{d t}-\frac{d N_{2}}{d t}\right)=\frac{d n}{d t}
$$

On the other hand, the current intensity remaining constant, from eq 3

$$
\frac{d^{2} N_{1}}{d y d t}+\frac{d^{2} N_{1}}{d y d t}=0
$$

and this together with eq 4 gives

and

$$
\frac{d^{2} N_{1}}{d y d t}=\frac{d n}{d t}
$$

$$
\frac{d^{2} N_{2}}{d y d t}=-\frac{d n}{d t}
$$

Differentiating eq 1 and 2 with respect to $y$ and using the relations just given,

and

$$
\frac{d n}{d t}=U_{1} \frac{d}{d y}\left(n \frac{d P}{d y}\right)+k_{1} \frac{d^{2} n}{d y^{2}},
$$

$$
\frac{d n}{d t}=-U_{2} \frac{d}{d y}\left(n \frac{d P}{d y}\right)+k_{2} \frac{d^{2} n}{d y^{2}} .
$$

Multiply the first of these by $U_{2}$, the second by $U_{1}$, and adding the resulting equations, one obtains

$$
\frac{d n}{d t}=\frac{k_{2} U_{1}+k_{1} U_{2}}{U_{1}+U_{2}} \frac{d^{2} n}{d y^{2}}
$$

Now the form of this equation is exactly the form of the diffusion equation of an ordinary nonelectrolytic solution. Hence, the changes in the concentrations of an electrolyte proceed with the diffusitivity constant

$$
D=\frac{k_{2} U_{1}+k_{1} U_{2}}{U_{1}+U_{2}}
$$

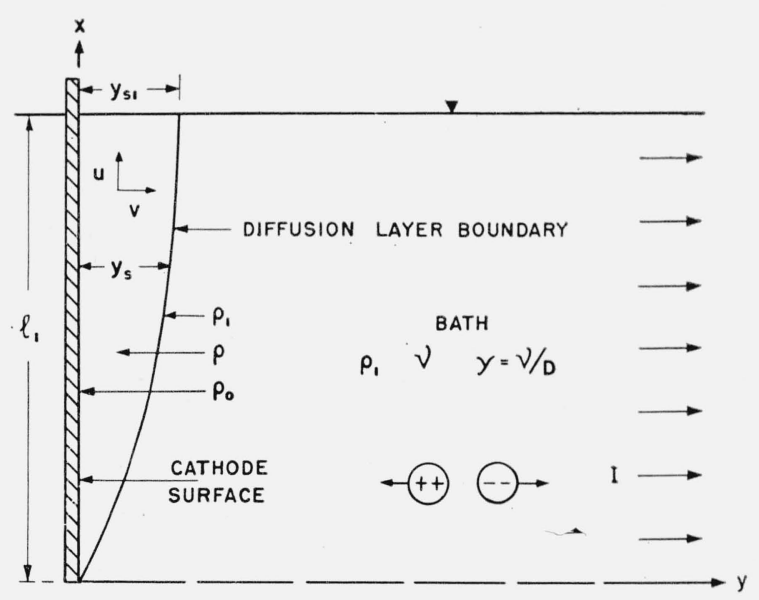

Figure 1. Geometrical and physical quantities relating to the diffusing layer.

As this relation holds good however slight the electric current may be, it may be supposed that the relation holds in the limit when the current is nil. Therefore, $D$ is the ordinary coefficient of diffusion of the solution, and thus a relation exists connecting this coefficient with the ionic quantities, that is, with the specific mobilities and the coefficients of diffusion of the two ions.

If the relations

$$
k_{1}=C U_{1} \text { and } k_{2}=C U_{2}
$$

exist, where $C$ is a constant independent of the concentration of the ions, then

$$
D=\frac{2 U_{1} U_{2}}{U_{1}+U_{2}} C
$$

Now, from the theory of electrolytic diffusion by Nernst [4] we have

$$
D=\frac{2 U_{1} U_{2}}{U_{1}+U_{2}} R T,
$$

where $T$ is the absolute temperature and $R$ the gas constant. Accordingly, the constant $C$ in eq 13 may be identified with the product $R T$ in eq 14 .

\section{The Equations of Motion and of Diffusion}

Taking the case of a plane cathode surface, held vertically, a point at the lower edge may be chosen as the origin of the rectangular coordinate system $(x, y)$ (see fig. 1). The positive $x$ axis is drawn vertically upward and the positive $y$ axis normal to the surface and into the electrolyte body. Denoting the velocity components along the $x$ and the $y$ axes by $u$ and $v$, respectively, the equations of motion are

$$
u \frac{\partial u}{\partial x}+v \frac{\partial u}{\partial y}=-\frac{1}{\rho} \frac{\partial p}{\partial x}-g+\nu \frac{\partial^{2} u}{\partial y^{2}},
$$




$$
0=-\frac{1}{\rho} \frac{\partial p}{\partial y}
$$

where $p$ is the pressure, $\nu$ the kinematic viscosity of the solution, $\rho$ the density, and $g$ the constant of gravitational acceleration. These are the equations of motion used in viscous boundary layer theory.

The pressure must be expressed in terms of the densities. Denoting the density of the electrolytic bath at points far removed from the cathode surface by $\rho_{1}$, the density near the surface may be expressed by the relation

$$
\rho=\rho_{1}-\Delta \rho,
$$

where $\Delta \rho$ is the deficiency of the density in the layer. The greatest reduction of density is at the cathode surface, that is at the plane $y=0$. Let the average value of $\Delta \rho$ over the entire length of the cathode surface be denoted by $\Delta \rho_{m}$. The study of the densities in the layer is done conveniently, as will be seen later, by considering the ratio

$$
\alpha=\frac{\Delta \rho}{\Delta \rho_{m}} .
$$

Another quantity important for the analysis is the effective gravity

$$
g \frac{\Delta \rho_{m}}{\rho_{1}}=G .
$$

Now, the second boundary layer equation, eq 16 , states that in the layer next to the cathode surface the pressure along a horizontal plane remains constant. Moreover, all motions being nil in the main body of the bath solution, the pressures are hydrostatic and hence

$$
p=p_{0}-\rho_{1} g x,
$$

where $p_{0}$ is the pressure at points in the horizontal plane passing through the origin. Thus,

$$
\frac{1}{\rho} \frac{\partial p}{\partial x}=-g \frac{\rho_{1}}{\rho} .
$$

Remembering that $\Delta \rho$ is a small quantity and making use of the relations in eq 18 and 19, we have

$$
-\frac{1}{\rho} \frac{\partial p}{\partial x}-g=G \alpha
$$

and thus eq 15 reduces to

$$
u \frac{\partial u}{\partial x}+v \frac{\partial u}{\partial y}=\nu \frac{\partial^{2} u}{\partial y^{2}}+G \alpha .
$$

This is the equation of motion that is appropriate to the problem at hand. To this must be added the conditions of continuity,

$$
\frac{\partial u}{\partial x}+\frac{\partial v}{\partial y}=0
$$

Next will be considered the equation of diffusion. In the presence of convective currents, the diffusion equation takes the form

$$
\frac{\partial n}{\partial t}+u \frac{\partial n}{\partial x}+v \frac{\partial n}{\partial y}=D\left(\frac{\partial^{2} n}{\partial x^{2}}+\frac{\partial^{2} n}{\partial y^{2}}\right),
$$

where $n$ is the number of particles of the diffusing material per unit volume. This relation reduces to Fick's equation when convective currents are absent. Although the equation is well known, it may be helpful to give a derivation in order to clarify the ideas involved. Neither the name of the investigator giving the first derivation nor the manner of derivation is known to us.

Consider a parallelepiped of sides $d x, d y$, and 1 . Let the number of particles crossing the face at $x$ perpendicular to the $x$ axis during time $d t$ be

$$
N_{11} d y d t, \quad N_{11}=-D \frac{\partial n}{\partial x}+n u,
$$

and the face at $x+d x$ be

$$
N_{12} d y d t=\left(N_{11}+\frac{\partial N_{11}}{\partial x} d x\right) d y d t .
$$

Similarly, let the number of particles crossing the face at $y$ perpendicular to the $y$ axis be

$$
N_{21} d x d t, \quad N_{21}=-D \frac{\partial n}{\partial y}+n v
$$

and the face at $y+d y$ be

$$
N_{22} d y d t=\left(N_{21}+\frac{\partial N_{21}}{\partial y} d y\right) d x d t
$$

so the accumulation of the particles in the parallelepiped is

$$
-\left(\frac{\partial N_{11}}{\partial x}+\frac{\partial N_{21}}{\partial y}\right) d x d y d t
$$

But the accumulation is also

$$
\frac{\partial n}{\partial t} d x d y d t
$$

Hence,

or

$$
\frac{\partial n}{\partial t}=-\frac{\partial N_{11}}{\partial x}-\frac{\partial N_{21}}{\partial y}
$$

$$
\frac{\partial n}{\partial t}=D\left(\frac{\partial^{2} n}{\partial x^{2}}+\frac{\partial^{2} n}{\partial y^{2}}\right)-u \frac{\partial n}{\partial x}-v \frac{\partial n}{\partial y}-n\left(\frac{\partial u}{\partial x}+\frac{\partial v}{\partial y}\right) .
$$

The last term in the right-hand number vanishes because of the incompressibility of the solution. This now proves the general equation of diffusion given by eq 25. A tacit assumption of the derivation is that the ratio of the volume of the diffusive 
particles to the volume of the solution is a small fraction. Stated otherwise, the result is valid for small concentrations.

The diffusion equation is much simplified when applied to the cathode film boundary layer. First, the concentrations do not vary appreciably with time. Second, the layer being very thin and comparatively very long, the quantity $\partial^{2} n / \partial x^{2}$ is small in comparison with $\partial^{2} n / \partial y^{2}$. Accordingly, eq 25 becomes

$$
u \frac{\partial n}{\partial x}+v \frac{\partial n}{\partial y}=D \frac{\partial^{2} n}{\partial y^{2}}
$$

and since $n$ is proportional to $\alpha$, also

$$
u \frac{\partial \alpha}{\partial x}+v \frac{\partial \alpha}{\partial y}=D \frac{\partial^{2} \alpha}{\partial y^{2}}
$$

This is the diffusion equation appropriate to the problem at hand. Equations 23, 24, and 26 govern the movement of liquid and the distribution of the densities in the cathode film. subject to the boundary conditions.

\section{Boundary Conditions}

The boundary conditions to be considered are of two kinds. One kind relates to the velocities and the other to the densities, the latter having electrochemical implications.

The boundary conditions to be assigned to velocity components are the most obvious ones. The solution or the liquid of the film being viscous, $u$ and $v$ vanish at the cathode surface; that is

$$
u=v=0, \quad y=0 .
$$

To state the condition for the velocities at large distances away from the cathode surface it is necessary to introduce first the concept of the boundary layer thickness (see fig. 1). The boundary layer thickness, $y_{s}$, is such that the density of the solution at this distance from the cathode differs only imperceptibly from the density of the bath. Since the movement of liquid in general is nil in the main portion of the bath, a second condition for the velocities is

$$
u=0, \quad y=y_{s} .
$$

It is obvious that the thickness $y_{s}$ of the boundary layer at the lower edge of the cathode surface, that is at $x=0$, vanishes. 'This means that the liquid moving upward in the film, must be replenished by a later and horizontal movement of the bath solution into the film region. Hence

$$
v>0, \quad y=y_{s} .
$$

The assumption of vanishing velocities in the bath in the vertical direction is an idealization. Under the actual conditions of experimentation, arbitrary movements in the bath may be present even when forced agitation is not resorted to. Since the movement of liquid in the film next to the cathode surface is upwald and in the film. next to the anode downward, it is obvious that a circulation is induced in the bath liquid. Due to accidental variations of temperature at places the regularity of the circulatory motion will be disturbed and will give rise to weak eddying motions. These will be most noticeable near the upper surface of the bath and close to the cathode. Such accidental disturbances can be imagined to have only minor effects on the main motion in the film and thus will be ignored.

The boundary conditions that are to be assigned to the densities are not obvious, especially the one relating to the value of the densities at the cathode surface. Regarding this, it seems that two different statements may be made depending on whether the transmitted electric current is of the limiting value or is inferior to this limiting value. Taking the case of weak electric currents, we may consider the question of the densities at the cathode surface as follows:

Assume that the ionic system is binary and ionization is complete. Since at the cathode surface, that is at $y=0, d N_{2} / d t=0$, eq 3 gives

$$
\frac{d N_{1}}{d t}=\frac{I}{s e},
$$

and thus eq 1 and 2 now become

$$
\frac{I}{s e}=n U_{1} \frac{d P}{d y}+k_{1} \frac{d n}{d y},
$$

and

$$
0=n U_{2} \frac{d P}{d y}-k_{2} \frac{d n}{d y}
$$

where $n$ is the number of ions of each kind per unit volume. Eliminating the potential gradient between these two equations and making use of eq 11,

$$
D \frac{d n}{d y}=\frac{U_{2}}{U_{1}+U_{2}} \frac{I}{s e} .
$$

This is the relation between the electric current intensity and the gradient of $n$ at the cathode surface.

It is desirable that the quantity $n$ in eq 30 be replaced by $c$, the latter being defined as the gram equivalent of the salt per cubic centimeter of the solution. In a unit cube there are $n$ cations and $n$ anions each of ionic valence $s$, and the system is completely ionized. Introducing $N$, the Avogadro number, the molar concentration is $n / N$ and hence the concentration in gram equivalent of the salt per cubic centimeter of the solution is

$$
c=\frac{n s}{N},
$$

and eq 30 now becomes

$$
D \frac{d c}{d y}=\frac{U_{2}}{U_{1}+U_{2}} \cdot \frac{I}{e N}
$$


Since $e N$ is the universal constant $F$, with the numerical value $F=96450$, and with the dimensions coulomb per gram equivalent,

$$
D \frac{d c}{d y}=\frac{U_{2}}{U_{1}+U_{2}} \cdot \frac{I}{F}
$$

The same condition may be expressed in terms of densities instead of concentrations. For the majority of electrolyte solutions the variation of densities with the concentration is linear; that is

$$
\frac{d c}{d \rho}=m
$$

For copper sulphate $m=1.32 \times 10^{-2}$ gram equivalent per gram; and for nickel chloride $m=1.71 \times 10^{-2}$ gram equivalent per gram. Equation 32 may now be written as

$$
m D \frac{d \rho}{d y}=\frac{U_{2}}{U_{1}+U_{2}} \frac{I}{F}, \quad y=0 .
$$

This is the relation between the intensity of the electric current and the gradient of the density at a point of the cathode surface. It is the only relation that may be derived on the basis of simple concepts of an electrochemical nature. Accordingly, we are not certain of the state of the densities at the cathode surface for the present.

Two simplifying assumptions may be made. First, it may be supposed that the current intensity is uniform at the cathode surface. The resulting boundary condition would be that the density gradient is uniform at the cathode surface. Second, it may be supposed that the density is constant at the cathode surface. For the present analysis we shall adopt the second alternative simply for the reason that the corresponding analysis may be completed with ease. Thus it will be supposed that,

and

$$
\Delta \rho=\Delta \rho_{m}, \quad y=0,
$$

In view of eq 18

$$
\Delta \rho=0, \quad y=\infty .
$$

and

$$
\alpha=1, \quad y=0,
$$

$$
\alpha=0, \quad y=\infty .
$$

The set of equations $27,28,35$, and 36 constitute the boundary conditions to be considered in solving the set of equations 23, 24, and 26.

Since the relation implied in eq 34 is valid for all conditions, it may be used to consider average effects. Expressing $\rho$ in terms of $\alpha$ through the use of eq 18, multiplying the two sides of the resulting equation by $d x$, and integrating between $x=0$ and $x=l_{1}$, where $l_{1}$ is the length of immersion of the cathode surface,

$$
-m D \Delta \rho_{m} \int_{0}^{l_{1}}\left(\frac{d \alpha}{d y}\right) d x=\frac{U_{2}}{U_{1}+U_{2}} \frac{I l_{1}}{F}
$$

where now $I$ is the average intensity of the electric current measured in amperes per square centimeter. We shall use this relation to compute the transport number of copper ions taking our data from the results of the Brenner experiments.

\section{The Dimensional Form of the Basic Equations}

The solution of the basic equations subject to the boundary conditions indicated above will be greatly simplified if the equations are changed into forms involving dimensionless quantities only. The clue to the proper course of analysis may be found in the method of Blasius for treating the viscous boundary layer of plates in a current of air.

The limiting values of the densities and the velocities being uniform at the boundaries of the cathode film or of the diffusion layer, it is to be expected that the distribution of the velocities and of the density reductions in different normal sections are affine to each other. The affinities will be established by the introduction of the characteristic length $\delta$ and the characteristic velocity $U$, both of which are functions of $x$ only.

Introducing the stream function $\psi$, such that

$$
u=\frac{\partial \psi}{\partial y}, \quad v=-\frac{\partial \psi}{\partial x},
$$

the condition of incompressibility, eq 24, is automatically satisfied. In terms of the stream function the equation of motion, eq 23, and the equation of diffusion, eq 26, transform to

$$
\frac{\partial \psi}{\partial y} \cdot \frac{\partial^{2} \psi}{\partial y \partial x}-\frac{\partial \psi}{\partial x} \cdot \frac{\partial^{2} \psi}{\partial y^{2}}=\nu \frac{\partial^{3} \psi}{\partial y^{3}}+G \alpha,
$$

and

$$
\frac{\partial \psi}{\partial y} \cdot \frac{\partial \alpha}{\partial x}-\frac{\partial \psi}{\partial x} \cdot \frac{\partial \alpha}{\partial y}=D \frac{\partial^{2} \alpha}{\partial y^{2}}
$$

Introducing the new variables

$$
\eta=y / \delta,
$$

and

$$
H=\frac{\psi}{U \delta},
$$

and remembering that $U$ and $\delta$ are functions of $x$ only, we have, first

$$
\frac{\partial}{\partial y}=\frac{\partial \eta}{\partial y} \cdot \frac{d}{d \eta}=\frac{1}{\delta} \frac{d}{d \eta},
$$

and

$$
\frac{\partial}{\partial x}=\frac{\partial \eta}{\partial x} \frac{d}{d \eta}=-\frac{\eta}{\delta} \frac{d \delta}{d x} \frac{d}{d \eta},
$$

and hence

$$
\frac{\partial \psi}{\partial x}=H \frac{d}{d x}(U \delta)-U \frac{d \delta}{d x} \eta \frac{d H}{d \eta},
$$




$$
\begin{gathered}
\frac{\partial \psi}{\partial y}=U \frac{d H}{d \eta} \\
\frac{\partial^{2} \psi}{\partial x \partial y}=\frac{d U}{d x} \frac{d H}{d \eta}-\frac{U \eta}{\delta} \frac{d \delta}{d x} \frac{d^{2} H}{d \eta^{2}} \\
\frac{\partial^{2} \psi}{\partial y^{2}}=\frac{U}{\delta} \frac{d^{2} H}{d \eta^{2}}
\end{gathered}
$$

and

$$
\frac{\partial^{3} \psi}{\partial y^{3}}=\frac{U}{\delta^{2}} \frac{d^{3} H}{d \eta^{3}}
$$

Substituting these expressions in eq 39 and 40 ,

$-U \frac{d U}{d x}\left(\frac{d H}{d \eta}\right)^{2}+\frac{U}{\delta} \frac{d}{d x}(U \delta) \cdot H \frac{d^{2} H}{d \eta^{2}}+\nu \frac{U}{\delta^{2}} \frac{d^{3} H}{d \eta^{3}}+G \alpha=0$,

and

where

$$
\frac{\gamma}{\delta} \frac{d}{d x}(U \delta) H \frac{d \alpha}{d \eta}+\frac{\nu}{\delta^{2}} \frac{d^{2} \alpha}{d \eta^{2}}=0
$$

$$
\gamma=\frac{\nu}{D}
$$

Now eq 50 and 51 will be freed of the differential operators involving $x$ if one can write

$$
M_{1} U \frac{d U}{d x}=M_{2} \frac{U}{\delta} \frac{d}{d x}(U \delta)=\frac{\nu U}{\gamma \delta^{2}}=G
$$

and

$$
\frac{M_{2}}{\delta} \frac{d}{d x}(U \delta)=\frac{\nu}{\gamma \delta^{2}}
$$

where $M_{1}$ and $M_{2}$ are numerical constants. In view of these provisional relations, the two equations simplify to

$$
\gamma \frac{d^{3} H}{d \eta^{3}}+\frac{1}{M_{2}} H \frac{d^{2} H}{d \eta^{2}}-\frac{1}{M_{1}}\left(\frac{d H}{d \eta}\right)^{2}+\alpha=0,
$$

and

$$
\frac{H}{M_{2}} \frac{d \alpha}{d \eta}+\frac{d^{2} G}{d \eta^{2}}=0
$$

In fact, the provisional equations 53 and 54, determine the dependence of the characteristic velocity $U$ and the characteristic length $\delta$ on the variable $x$. The provisional equations 53 and 54 imply

$$
\begin{aligned}
& M_{1} \delta \frac{d U}{d x}=M_{2} \frac{d}{d x}(U \delta), \\
& M_{2} \delta \frac{d}{d x}(U \delta)=\frac{\nu}{\gamma},
\end{aligned}
$$

and

$$
\nu U=\gamma \delta^{2} G \text {. }
$$

It is now obvious that $U$ and $\delta$ each are single power terms of $x$. Assuming

$$
\begin{gathered}
U=A x^{r}, \\
\delta=B x^{s},
\end{gathered}
$$

where $A$ and $B$ are constants involving the physical characteristics of the electrolytic solution. Substituting these expressions in eq 57, 58, and 59, comparing the powers of $x$ and the coefficients for the individual equations, the comparisons yield

$$
\begin{gathered}
M_{1} r=M_{2}(s+r), \\
M_{2}(s+r) A B^{2}=\nu / \gamma \\
2 s+r-1=0, \\
r=2 s,
\end{gathered}
$$

and

$$
\nu A=\gamma B^{2} G \text {. }
$$

Equations 64 and 65 , yield $r=1 / 2$ and $s=1 / 4$. Equation 62 now yields

$$
2 M_{1}-3 M_{2}=0
$$

For simplicity subject to the last derived equations, we may put $M_{1}=1 / 2$ and $M_{2}=1 / 3$.

Substituting the values of the constants $A$ and $B$ that are obtained from eq 63 and 66 into eq 60 and 61 , there results

and

$$
U=2 G^{1 / 2} x^{1 / 2},
$$

$$
\delta=\left(\frac{4 \nu^{2}}{G \gamma^{2}}\right)^{1 / 4} x^{1 / 4}
$$

which are the functions showing the dependence of the characteristic velocity $U$ and the characteristic length $\delta$ on the independent variable $x$.

Substituting the values of $M_{1}$ and $M_{2}$ as found above into eq 55 and 56 , these simplify into

$$
\gamma \frac{d^{3} H}{d \eta^{3}}+3 H \frac{d^{2} H}{d \eta^{2}}-2\left(\frac{d H}{d \eta}\right)^{2}+\alpha=0,
$$

and

$$
\frac{d^{2} \alpha}{d \eta^{2}}+3 H \frac{d \alpha}{d \eta}=0
$$

which are the equation of motion and the equation of diffusion expressed in dimensionless forms.

In terms of the new variables, the velocity components $u$ and $v$ are, using eq 38,45 , and 46 ,

$$
u=U \frac{d H}{d \eta},
$$

and

$$
-v=\frac{d}{d x}(U \delta) H-U \frac{d \delta}{d x} \eta \frac{d H}{d \eta},
$$


and the boundary conditions are

$$
\left.\begin{array}{rl}
H=0, \quad \frac{d H}{d \eta}=0, & \eta=0, \\
\frac{d H}{d \eta}=0, & \eta=\infty, \\
\alpha=1, & \eta=0, \\
\alpha=0, & \eta=\infty .
\end{array}\right\}
$$

The two equations 69 and 70 become the basic equations for the problem of temperature variation of a heated plate placed vertically in a cool air bath, when

$$
\alpha=\frac{T-T_{1}}{T_{0}-T_{1}}
$$

where $T$ is the temperature near the plate, $T_{0}$ the temperature of the plate, and $T_{1}$ that of the cool air, and the parameter $\gamma$ is replaced by

$$
\sigma=\nu / k,
$$

where $k$ is the thermometric conductivity.

Goldstein [5] has discussed the Pohlhausen solution of the heated plate problem and the equations considered are similar to eqs 69 and 70 , when allowances are made for the variations in some of the coefficients. Thus, in the two basically different phenomena of the diffusion layer near a cathode plate and of the conduction layer near a heated plate, the corresponding parameters $\gamma$ and $\sigma$ play identical roles. The latter is well known as the Prandtl number. The former will be called the diffusion parameter for the present, since it is a dimensionless number expressing the ratio of the kinematic viscosity to the coefficient of diffusion. In forming the numerical value of $\gamma$, both $\mathrm{D}$ and $\nu$ must be expressed in square centimeters per second.

\section{An Approximate Solution of the Basic Equations}

Since an approximate solution is adequate for the present objectives, it is hardly necessary to effect a rigorous solution of the two equations, 69 and 70 . To prepare the way for the approximate solution, it is necessary to introduce the boundary layer thickness $y_{s}$, which is a function of $x$. To the distances $y$ and $y_{s}$ correspond the parametric distances $\eta$ and $\eta_{s}$, such that

$$
\eta=y / \delta \text { and } \eta_{s}=y_{s} / \delta .
$$

We introduce the ratio

$$
\theta=\eta / \eta_{s}=y / y_{s}
$$

It is obvious that the quantity $\theta$ will serve as the independent variable in the place of $\eta$. Indeed, the basic equations 69 and 70 transform to

$$
\gamma \frac{d^{3} H}{d \theta^{3}}+3 \eta_{s} H \frac{d^{2} H}{d \theta^{2}}-2 \eta_{s}\left(\frac{d H}{d \theta}\right)^{2}+\eta_{s}^{3} \alpha=0,
$$

and

$$
\frac{d^{2} \alpha}{d \theta^{2}}+3 \eta_{s} H \frac{d \alpha}{d \theta}=0
$$

with the boundary conditions

$$
\left.\begin{array}{rlrl}
H & =0, & & \theta=0, \\
\frac{d H}{d \theta} & =0, & & =0, \\
\frac{d H}{d \theta} & =0, & & =1, \\
\alpha & =1, & \theta & =0, \\
\alpha & =0, & & \theta=1,
\end{array}\right\}
$$

in place of the set shown by eq 73 .

It is obvious, intuitively, that the space rate of change of the velocity components, $\partial u / \partial y$ and $\partial v / \partial y$, each vanish at large distances from the cathode surface. These conditions are fulfilled likewise quite approximately at distances equaling or exceeding $y_{s}$. This observation suggests the additional boundary conditions

$$
\left.\begin{array}{ll}
\frac{d^{2} H}{d \theta^{2}}=0, & \theta=1, \\
\frac{d \alpha}{d \theta}=0, & \theta=1,
\end{array}\right\}
$$

the first of these resulting from eq 48. All the boundary conditions of eqs 80 and 81 are satisfied if it is assumed that

$$
\alpha=1-\sin \frac{\pi}{2} \theta,
$$

and

$$
H=\frac{A_{1}}{\pi}\left[\frac{5}{4}-\cos \pi \theta-\frac{1}{4} \cos 2 \pi \theta\right] .
$$

The above analysis involves the quantities $A_{1}$ and $\eta_{s}$, which at present are not known. For the determination of their values the differential equations 78 and 79 are at our disposal. Multiplying these equations by $d \theta$, integrating between the limits $\theta=0$ and $\theta=1$, and making use of the boundary conditions, the result is

$$
-\gamma\left(\frac{d^{2} H}{d \theta^{2}}\right)_{0}-5 \eta_{s} \int_{0}^{1}\left(\frac{d H}{d \theta}\right)^{2} d \theta+\eta_{s}^{3} \int_{0}^{1} \alpha d \theta=0,
$$


and

$$
\left(\frac{d \alpha}{d \theta}\right)_{0}+3 \eta_{s} \int_{0}^{1} \alpha \frac{d H}{d \theta} d \theta=0
$$

after resorting to the rules of integration by parts. Introducing the values of $\alpha$ and $H$ from eq 82 and 83 and effecting the integrations, we find

$$
\begin{aligned}
& -2 \pi \gamma A_{1}-\frac{25}{8} \eta_{s} A_{1}^{2}+\eta_{s}^{3}\left(\frac{\pi-2}{\pi}\right)=0, \\
& -\frac{\pi}{2}+\frac{14}{5} \eta_{s} \frac{A_{1}}{\pi}=0
\end{aligned}
$$

and hence

and

$$
\eta_{s}^{4}=\frac{5 \pi^{4}}{14(\pi-2)}\left(\gamma+\frac{125}{448} \pi\right)
$$

$$
A_{1}=\frac{5 \pi^{2}}{28 \eta_{s}} .
$$

The expressions for $\eta_{s}$ and $A_{1}$ as given by eq 86 and 87, respectively, and the expressions for $\alpha$ and $H$ as given by eq 82 and 83 , respectively, constitute the first approximate solution of the diffusion layer problem based on the assumption that the reduction of densities, or concentrations, at the cathode surface is uniform.

\section{Thickness of the Cathode Diffusion Layer}

Inasmuch as the diffusion parameter $\gamma=\nu / D$ is ordinarily a very large quantity, it is sufficient to write in the place of eq 86

$$
\eta_{s}^{4}=\frac{5 \pi^{4} \gamma}{14(\pi-2)} .
$$

Introducing the value of $y_{s}$ from eq 76 and the value of $\delta$ from eq 68 , we find first

$$
y_{s}=\left[\frac{5 \pi^{4} \gamma}{14(\pi-2)}\right]^{1 / 4}\left[\frac{4 \nu^{2}}{G \gamma^{2}}\right]^{1 / 4} x^{1 / 4}
$$

and next introducing the value of $G$ from eq 19

$$
y_{s}=3.33\left(\frac{\rho_{1} \nu^{2}}{g \Delta \rho_{m} \gamma}\right)^{1 / 4} x^{1 / 4}
$$

This is the expression that gives the thickness of the diffusion layer along the cathode surface (See fig. 2). The thickness is not uniform, but increases from the zero value at the lower edge of the cathode surface to a maximum at the upper end of the cathode surface still in contact with the electrolyte. The rate of increase of the thickness, however, is very gradual due to the fact that the thickness if proportional to the one fourth power of $x$, the distance from the lower end of the cathode. Again at a chosen point $x$ the thickness of the layer

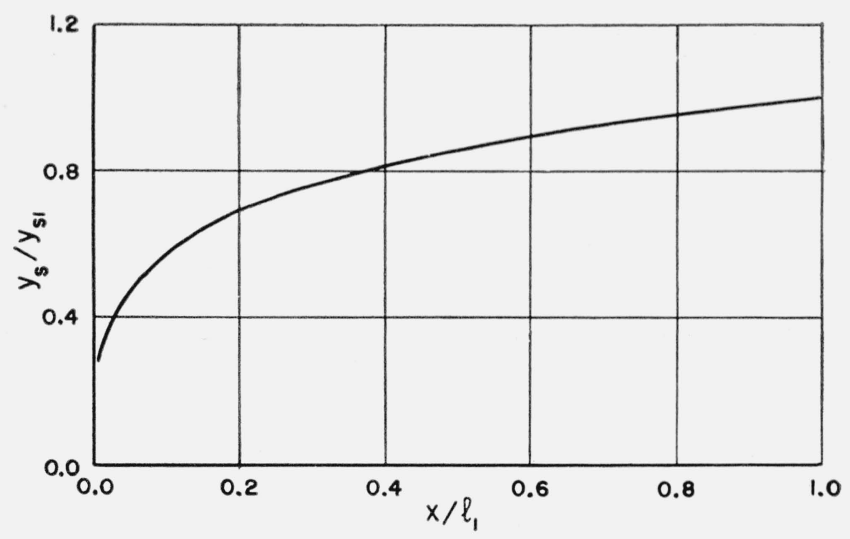

FIGURE 2. Shape of the boundary of the diffusion layer.

decreases when $\Delta \rho_{m}$, the maximum reduction of the density at the cathode surface, is increased. Since the decrease of density at the cathode surface is proportional to the electrical current density, when the latter is inferior to its limiting value, it is to be expected that a decrease in the intensity of current will cause the thickness of the diffusion layer to be augmented.

Having established the magnitude of the diffusion layer thickness, it is of interest to inquire next into the normal density gradient at the cathode surface. The variation of the density gradient will be best understood if it is expressed in terms of the average density gradient, the averaging being made over the entire cathode surface. In view of eq 18, the quantity

$$
\left(\frac{\partial \alpha}{\partial y}\right)_{0} \sqrt{\left(\frac{\partial \alpha}{\partial y}\right)_{0}}=R
$$

where the numerator on the left-hand side refers to local values at the point $x$ and the denominator to the average values, is the ratio to be considered. Since

$$
\frac{\partial \alpha}{\partial y}=\frac{\partial \alpha}{\partial \theta} \cdot \frac{\partial \theta}{\partial y}
$$

we have from eq 77 and 82

$$
\left(\frac{\partial \alpha}{\partial y}\right)_{0}=-\frac{\pi}{2 y_{s}}
$$

or

$$
\left(\frac{\partial \alpha}{\partial y}\right)_{0}=-\frac{c_{1}}{x^{1 / 4}}
$$

where

$$
c_{1}=\frac{\pi}{6.66}\left(\frac{\rho_{1} \nu^{2}}{g \Delta \rho_{m} \gamma}\right)^{-1 / 4}
$$

Accordingly, the average value over the length $l_{1}$ of the cathode surface is

$$
\overline{\left(\frac{\partial \alpha}{\partial y}\right)_{0}}=-\frac{4}{3} \frac{c_{1}}{l_{1}^{1 / 4}}
$$


and the desired ratio is

$$
R=\frac{3}{4}\left(\frac{l_{1}}{x}\right)^{1 / 4}
$$

which gives the proportionate variation of the normal density gradient over the cathode surface The variation is shown graphically in figure 3 . In the section of the cathode from $x / l_{1}=0.2$ to $x / l_{1}=1.0$ the ratio is nearly constant. This circumstance assures the utility of the present solutions even for the case in which the normal concentration gradient is strictly constant over the entire surface of the cathode, provided that one deals with the average values of the densities in the planes close and parallel to the cathode surface.

If the diffusion layer thickness at $x=l_{1}$, that is at the upper end of the cathode surface just in contact with the bath, be denoted by $y_{s 1}$, then the average value of the normal density gradient, expressed in terms of the maximum density reduction, is

$$
\overline{\left(\frac{\partial \alpha}{\partial y}\right)_{0}}=-\frac{2.1}{y_{s 1}} \text {. }
$$

Use will be made, subsequently, of this relation.

\section{Velocitus in the Diffusion Layer}

Consider first the velocity component $u$, that is the component parallel to the surface of the cathode. Now,

$$
U=2 G^{1 / 2} x^{1 / 2}
$$

where

$$
u=U \frac{d H}{d \eta}
$$

Since

$$
\frac{d H}{d \eta}=\frac{d H}{d \theta} \cdot \frac{d \theta}{d \eta}
$$

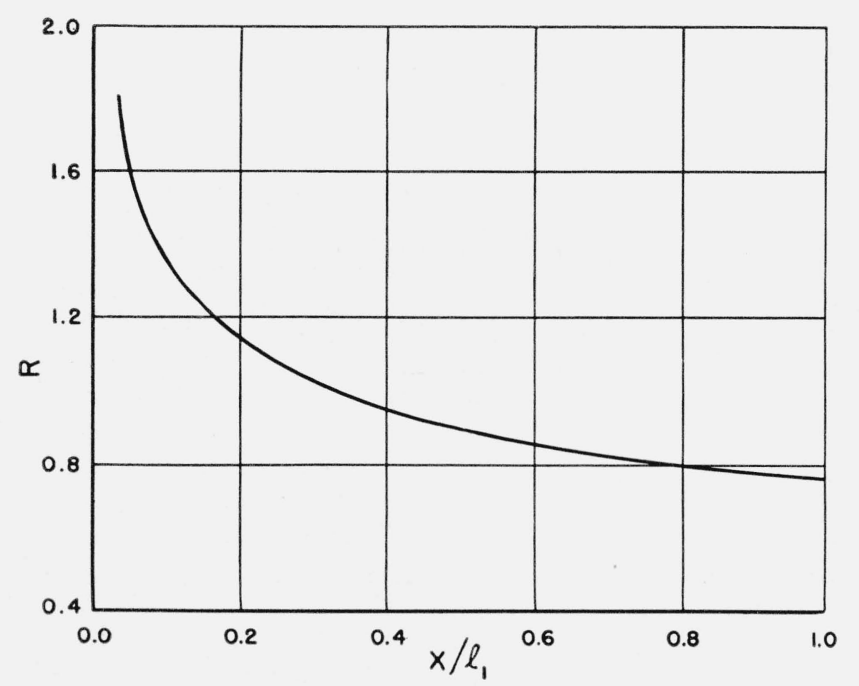

Figure 3. Variation of the density gradient along the cathode surface. eq 71 may serve, in view of eq 77,83 , and 87 , to give the distribution of the velocities in a normal section of the diffusion layer. There is, however, another method for representing the velocity distribution. Moving away from the cathode surface in a normal section, $u$ is zero at the cathode surface, increases to a maximum value $u_{m}$, and then decreases to zero at the limit of the boundary layer, that is at $y=y_{s}$. Thus, the distribution of the velocity may be obtained also by considering the ratio $u / u_{m}$. Now

$$
u_{m}=U\left(\frac{d H}{d \eta}\right)_{m} .
$$

In terms of the variable $\theta$,

$$
\left(\frac{d H}{d \eta}\right)_{m}=\frac{1}{\eta_{s}}\left(\frac{d H}{d \theta}\right)_{m} .
$$

If $\theta_{1}$ be the value of $\theta$ that makes $u$ a maximum, we have from eq 83 ,

$$
\eta_{s}\left(\frac{d H}{d \eta}\right)_{m}=A_{1}\left[\sin \pi \theta_{1}+\frac{1}{2} \sin 2 \pi \theta_{1}\right] .
$$

The value of $\theta_{1}=1 / 3$ satifies the relation.

This makes

$$
\cos \pi \theta_{1}+\cos 2 \pi \theta_{1}=0 .
$$

$$
\sin \pi \theta_{1}+\frac{1}{2} \sin 2 \pi \theta_{1}=1.299,
$$

and therefore

$$
\left(\frac{d H}{d \theta}\right)_{m}=1.299 A_{1}
$$

Substituting into eq 96 , from eq $98,97,88$, and 67 gives

or using eq 19 ,

$$
u_{m}=0.82\left(\frac{G}{\gamma}\right)^{1 / 2} x^{1 / 2}
$$

$$
\frac{u_{m}}{\sqrt{g x}}=0.82\left(\frac{\Delta \rho_{m}}{\rho_{1} \gamma}\right)^{1 / 2} .
$$

Accordingly, the maximum value of $u$ in a normal section changes from one section to another and increases with the one-half power of the distance from the lower end of the cathode.

Since,

$$
u / u_{m}=(d H / d \eta) /(d H / d \eta)_{m} .
$$

and introducing the relations in the equations 97 and 98 , the distribution of the velocity component $u$ in a normal section in the diffusion layer becomes

$$
u / u_{m}=0.77\left[\sin \pi \frac{y}{y_{s}}+\frac{1}{2} \sin 2 \pi \frac{y}{y_{s}}\right] .
$$




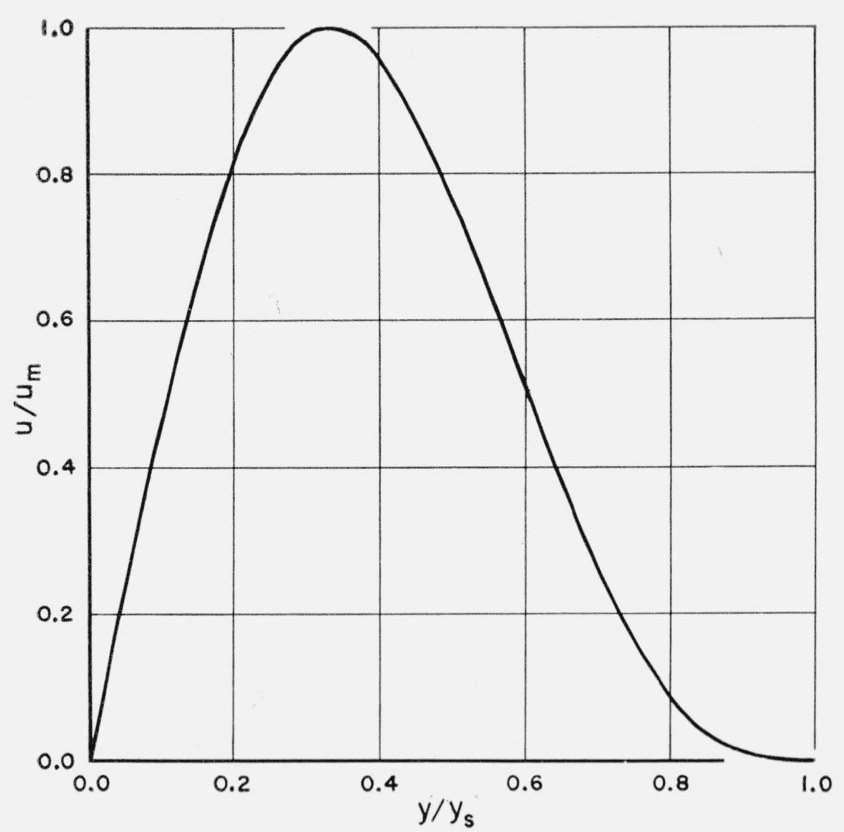

FiguRE 4. Distribution of the vertical velocity component.

The graphical representation of the distribution is given in figure 4 .

Next consider $v$, the velocity component normal to the cathode surface. The velocity can be written from eq 72 as

$$
v=-U \frac{d \delta}{d x}\left[3 H-\eta \frac{d H}{d \eta}\right]
$$

The magnitude of $v$ is largest at the liquid boundary of the diffusion layer. Let $v_{m}$ be this largest value. This occurs at $\eta=\eta_{s}$. Let $H_{s}$ be the value of $H$ at $\eta=\eta_{s}$ or at $\theta=1$. At $\eta=\eta_{s}, d H / d \eta=0$, and thus

$$
v_{m}=-3 \frac{d \delta}{d x} H_{s} U
$$

where

$$
H_{s}=2 A_{1} / \pi \text {. }
$$

In view of the values of $U$ and $\delta$ from. eq 67 and 68 ,

$$
U \frac{d \delta}{d x}=\frac{1}{2}\left(4 \nu^{2} G\right)^{1 / 4} x^{-1 / 4} \gamma^{-1 / 2}
$$

Substituting the above result in eq 102 and also introducing the value of $A_{1}$ from eq 87 ,

$$
v_{m}=-1.013\left(\nu^{2} G\right)^{1 / 4} \gamma^{-3 / 4} x^{-1 / 4} .
$$

Accordingly, the liquid of the bath moves horizontally into the cathode diffusion layer. The velocity with which the liquid approaches the cathode surface is variable along the length of the cathode surface, the maximum value occurring at the lower end of the cathode surface. After a certain elevation is reached the variation in the value of $v_{m}$ ceases to be pronounced.

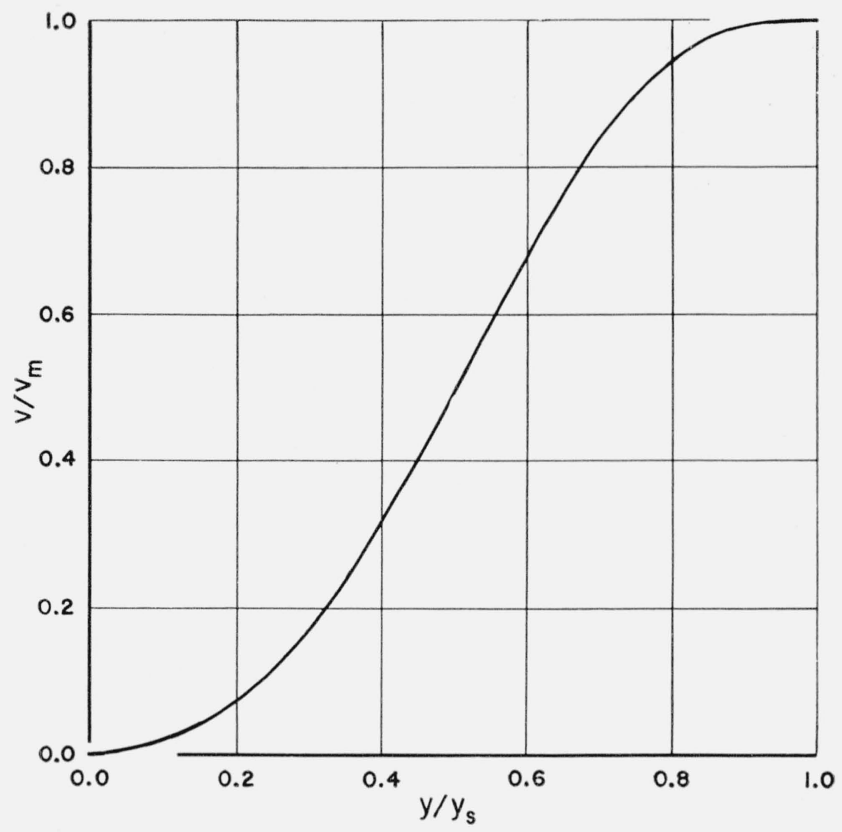

Figure 5. Distribution of the horizontal velocity component.

The representation of the distribution of $v$ in a normal section of the layer is most conveniently done by considering the ratio $v / v_{m}$. Now,

$$
\frac{v}{v_{m}}=\frac{1}{H_{s}}\left[H-\frac{\eta}{3} \frac{d H}{d \eta}\right]
$$

or in terms of $\theta$,

$$
\frac{v}{v_{m}}=\frac{1}{H_{s}}\left[H-\frac{\theta}{3} \frac{d H}{d \theta}\right]
$$

This gives, in view of eq 83 and 103,

$\frac{v}{v_{m}}=\frac{5}{8}-\frac{1}{2} \cos \pi \theta-\frac{1}{8} \cos 2 \pi \theta-\frac{\pi \theta}{6} \sin \pi \theta-\frac{\pi \theta}{12} \sin 2 \pi \theta$.

The graphical representation of the distribution is shown in figure 5 .

\section{The Brenner Freezing Method and the Laminar Mean Densities}

In the Brenner method [6] the electrolytic solution near a cylindrical cathode is suddenly frozen, successive layers of the solid of 0.003 - to 0.004 -inch thickness are carved off on a lathe, and the compositions of the lavers are determined. Thus, in this method the average concentration or the average density of a lamina of thickness $d y_{1}$, of distance $y_{1}$ from the cathode and of length $l_{1}$ are determined. Accordingly, if theoretical results are to be compared with the observed results of the Brenner experiments, it becomes necessary to derive a formula giving the average value of densities of a lamina of distance $y_{1}$ from the cathode surface, 


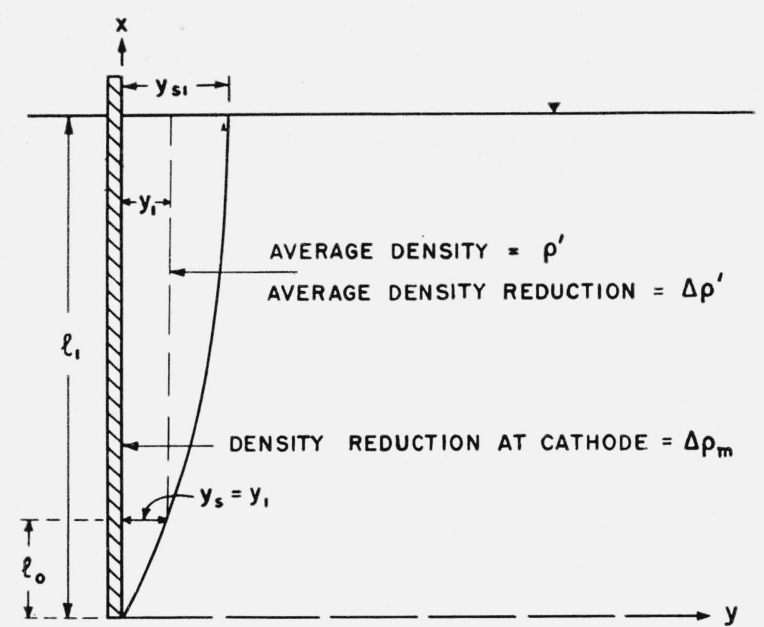

FIGURE 6. Geometrical and physical quantities relating to the diffusion layer in Brenner's tests.

Let the average density reduction in the lamina of length $l_{1}$ and of distance $y_{1}$ from the cathode surface be denoted by $\Delta \rho^{\prime}$. Mathematically,

$$
\Delta \rho^{\prime}=\frac{1}{l_{1}} \int_{0}^{l_{1}} \Delta \rho d x
$$

Let $l_{0}$ be the value of $x$ for which the boundary layer thickness equals $y_{1}$ (see fig. 6). Obviously, for values of $x<l_{0}, \Delta \rho=0$, and thus,

$$
\Delta \rho^{\prime}=\frac{1}{l_{1}} \int_{l_{0}}^{l_{1}} \Delta \rho d x
$$

Since, according to the present solutions, the density reduction at $y=y_{1}$ is given by the relation

$$
\Delta \rho=\Delta \rho_{m}\left(1-\sin \frac{\pi}{2} \frac{y_{1}}{y_{s}}\right)
$$

(from eq 82), then

$$
\Delta \rho^{\prime}=\Delta \rho_{m}\left[\frac{1}{l_{1}} \int_{l_{0}}^{l_{1}} d x-\frac{1}{l_{1}} \int_{l_{0}}^{l_{1}} \sin \frac{\pi}{2} \frac{y_{1}}{y_{s}} d x\right] .
$$

It must be remembered in effecting the integration of the second integral of the right-hand member, that $y_{1}$ is held constant. Putting

and

$$
y_{1} / y_{s}=\beta,
$$

$$
y_{1} / y_{s 1}=\beta_{1},
$$

It is seen from the law of the layer thickness, eq 89, that

$$
\begin{aligned}
& l_{1}=l_{0} / \beta_{1}^{4}, \\
& x=l_{0} / \beta^{4},
\end{aligned}
$$

and thus,

$$
d x=-4 l_{0} d \beta / \beta^{5} .
$$

Accordingly, in terms of the new variable $\beta$,

$$
\Delta \rho^{\prime}=\Delta \rho_{m}\left[1-\beta_{1}^{4}+4 \beta_{1}^{4} \int_{1}^{\beta_{1}} \sin \frac{\pi}{2} \beta \frac{d \beta}{\beta^{5}} .\right.
$$

For convenience, we may write

$$
F\left(\beta_{1}\right)=\beta_{1}^{4}-4 \beta_{1}^{4} \int_{1}^{\beta_{1}} \sin \frac{\pi}{2} \beta \frac{d \beta}{\beta^{5}}
$$

and hence,

$$
\Delta \rho^{\prime} / \Delta \rho_{m}=1-F\left(\beta_{1}\right) .
$$

Since $\beta_{1}=y_{1} / y_{s 1}$, we have also

$$
\Delta \rho^{\prime} / \Delta \rho_{m}=1-F\left(y_{1} / y_{s 1}\right) .
$$

This is the desired formula that will be used in analyzing the experimental data of Brenner. It involves the average reduction of density over a lamina, the average maximum reduction of density at the cathode surface, the distance of the lamina from the cathode, and the maximum thickness of the diffusion layer. The determination of the function $F\left(\beta_{1}\right)$ was carried out by replacing the term $\sin (\pi / 2) \beta$ appearing in the integrand by a power series in $\beta$ and then effecting the integration. The results of this determination are given in table 1 .

\section{An Analysis of the Results Obtained by Brenner with the Freezing Method}

The comparison between the results of the present analytical theory of the cathode diffusion layer and the results of the Brenner experiments on the reduction of the densities in the diffusion layer will

\begin{tabular}{|c|c|c|c|}
\hline$y_{1} / y_{s 1}$ & $F\left(y_{1} / y_{s 1}\right)$ & $y_{1} / y_{s 1}$ & $F\left(y_{1} / y_{s 1}\right)$ \\
\hline $\begin{array}{r}0 \\
0.1 \\
.2 \\
.3 \\
.4 \\
.5\end{array}$ & $\begin{array}{r}0.0000 \\
.2070 \\
.4024 \\
.5708 \\
.7099 \\
.8180\end{array}$ & $\begin{array}{r}0.6 \\
.7 \\
.8 \\
.9 \\
1.0\end{array}$ & $\begin{array}{r}0.8926 \\
.9388 \\
.9640 \\
.9796 \\
1.0000\end{array}$ \\
\hline
\end{tabular}
be carried out along the following lines. First, from the observed values of the reduction of density at the cathode surface, $\Delta \rho_{m}$, the maximum thickness of the diffusion layer, $y_{s 1}$, will be computed and compared with the observations. Second, using the same observed values of $\Delta \rho_{m}$, the ration $\Delta \rho^{\prime} / \Delta \rho_{m}$ will be computed for the distance $y_{1}$, and these will be compared with the observations. Third, $\Delta \rho_{m}$ will be computed from a consideration of the electric current intensities used in the expeiments and will be compared with the observations.

TABLE 1. Numerical values of the function $F\left(y_{1} / y_{s 1}\right)$

The Brenner [6] results relate to electrolytic baths of copper sulfate and nickel sulfate. The baths actually used were not pure binary systems, but this fact will be ignored. The complete data from the Brenner tests are collected in table 2. The 
TABLe 2. Diffusion layer densities of the Brenner test

\begin{tabular}{|c|c|c|c|c|}
\hline $\begin{array}{l}\text { Distance } \\
\text { from } \\
\text { cathode, } y_{1}\end{array}$ & $\begin{array}{l}\text { Normal- } \\
\text { ity, } \\
N\end{array}$ & $\begin{array}{c}\text { Density, } \\
\rho^{\prime}\end{array}$ & $\begin{array}{c}\text { Current } \\
\text { intensity, } \\
I\end{array}$ & $\underset{\substack{\text { Metallic } \\
\text { ion }}}{ }$ \\
\hline $\begin{array}{l}\quad c m \\
\text { 3. } 69 \times 10^{-2} \\
11.16 \\
\text { 18. } 79 \\
27.63 \\
37.63\end{array}$ & $\begin{array}{c}\text { Gram } \\
\text { equiv/liter } \\
1.274 \\
1.522 \\
1.800 \\
1.920 \\
1.931\end{array}$ & $\begin{array}{l}\mathrm{g} / \mathrm{cm}{ }^{3} \\
1.1009 \\
1.1174 \\
1.1381 \\
1.1465 \\
1.1473\end{array}$ & $a m p / c m^{2}$ & Copper \\
\hline $\begin{array}{r}3.83 \\
11.18 \\
21.05 \\
32.46 \\
44.12\end{array}$ & $\begin{array}{l}1.741 \\
1.885 \\
1.971 \\
1.985 \\
1.985\end{array}$ & $\begin{array}{l}1.132 \\
1.142 \\
1.149 \\
1.150 \\
1.150\end{array}$ & 0.01 & Nickel \\
\hline $\begin{array}{r}3.83 \\
12.36 \\
21.05 \\
30.00 \\
40.00\end{array}$ & $\begin{array}{l}\text { 1. } 561 \\
1.724 \\
1.872 \\
1.935 \\
1.959\end{array}$ & $\begin{array}{l}\text { 1. } 118 \\
\text { 1. } 130 \\
\text { 1. } 139 \\
\text { 1. } 146 \\
1.148\end{array}$ & 0.02 & Ajickel \\
\hline $\begin{array}{r}3.83 \\
12.36 \\
21.05 \\
30.00 \\
40.00\end{array}$ & $\begin{array}{l}\text { 1. } 382 \\
\text { 1. } 694 \\
1.900 \\
1.947 \\
1.981\end{array}$ & $\begin{array}{l}1.105 \\
1.128 \\
1.143 \\
1.147 \\
1.149\end{array}$ & 0.04 & Nickel \\
\hline
\end{tabular}

concentrations in the original data are in terms of the normalities. The densities as derived from the normality values are added. The average densities $\rho^{\prime}$ taken from the table are plotted against $y_{1}$ in figure 7 , and smooth curves are drawn through the points of observation. The extreme values $\rho_{0}$ and $\rho_{1}$ of the densities are read from the curves. The differences $\left(\rho_{1}-\rho_{0}\right)$ give $\Delta \rho_{m}$, the average maximum reduction of densities at the cathode surfaces. The difference $\left(\rho_{1}-\rho^{\prime}\right)$, where $\rho^{\prime}$ is the observed value of the densities corresponding to the distance $y_{1}$ gives $\Delta \rho^{\prime}$, the average reduction of the densities corresponding to the distance $y_{1}$ All these quantities, together with the ratio $\Delta \rho^{\prime} / \Delta \rho_{m}$, are entered in table 3.

From the observed values of $\Delta \rho_{m}$ and $\rho_{1}$ the maximum layer thickness, $y_{s 1}$, may be computed from the theoretical formula in eq 89. However, the use of eq 89 for this purpose requires that the parametric diffusion number, $\gamma$, be known. This number involves the ratio of the kinematic viscosity $\mu$, to the diffusion constant $D$, for each of the two electrolytic solutions. The viscosities of the electrolytes may be determined from the normalities using the Arrhenius [7] formula,

$$
\mu=\mu_{1}^{N},
$$

where $\mu_{1}$ is the specific viscosity of a normal solution referred to the solvent at the same temperature, $\mu$ is the specific viscosity of the solution having the normality N. Wagner [8] gives $\mu_{1}=1.358$ for copper sulfate and $\mu_{1}=1.361$ for nickel sulfate at the temperature $25^{\circ} \mathrm{C}$. The normalities of the bath solutions in the Brenner tests being 2 and the densities about $\rho=1.151$, the kinematic viscosities of the two solutions are practically equal and so $\mu=0.0144$ $\mathrm{cm}^{2} / \mathrm{sec}$. Regarding the diffusion coefficient $D$, the Thovert [9] values for copper sulfate may be supposed to apply as well to nickel sulfate. For copper sulfate at a temperature of $17^{\circ} \mathrm{C}, N=1.95$, the diffusion coefficient is $D=2.66 \times 10^{-6} \mathrm{~cm}^{2} / \mathrm{sec}$. Hence the value

$$
\gamma=0.572 \times 10^{4}
$$

may be used for the two solutions. As regards the cathode immersion length $l_{1}$, we take it to be $10 \mathrm{~cm}$, this being the value communicated to the author orally by Brenner.

Using the above-mentioned values of the pertinent quantities the magnitudes of the maximum laver thickness were computed from eq 85, and these are shown in table 4 . Also there are given in the table the observed values of the same quantity as read from the curves in figure 7 . At instances equalling the maximum layer thicknesses the average densities equal the bath densities. There is some difficulty

\begin{tabular}{|c|c|c|c|c|c|c|c|}
\hline $\begin{array}{l}\text { Distance } \\
\text { from cath- } \\
\text { ode, } y_{1}\end{array}$ & $\begin{array}{l}\text { Reduction of } \\
\text { density at } \\
\text { cathode, } \Delta \rho_{m}\end{array}$ & $\begin{array}{l}\text { Reduction of } \\
\text { density in } \\
\text { layer, } \Delta \rho^{\prime}\end{array}$ & $\begin{array}{c}\text { Theoretical } \\
\text { maximum } \\
\text { layer thickness, } \\
y_{s 1}\end{array}$ & $\begin{array}{c}\text { Proportionate } \\
\text { density re- } \\
\text { duction, } \\
1-\Delta \rho^{\prime} / \Delta \rho_{m}\end{array}$ & $\begin{array}{l}\text { Proportionate } \\
\text { distance from } \\
\text { cathode, } y_{1} / y_{s 1}\end{array}$ & $\begin{array}{c}\text { Current } \\
\text { intensity, } \\
I\end{array}$ & $\begin{array}{l}\text { Metallic } \\
\text { ion }\end{array}$ \\
\hline $\begin{array}{l}\quad c m \\
0.00 \\
3.69 \times 10^{-2} \\
11.16 \\
\text { 18. } 79 \\
27.63 \\
37.63\end{array}$ & $\begin{array}{l}\mathrm{g} / \mathrm{c} \mathrm{cm} \mathrm{m}^{3} \\
0.061\end{array}$ & $\left\{\begin{array}{c}\mathrm{g} / \mathrm{c} \mathrm{cm^{3 }} \\
0.061 \\
.047 \\
.031 \\
.010 \\
.002 \\
.000\end{array}\right.$ & $\begin{array}{c}\mathrm{cm} \\
0.0302\end{array}$ & $\begin{array}{r}0.00 \\
.23 \\
.49 \\
.84 \\
.97 \\
1.00\end{array}$ & $\begin{array}{r}0.00 \\
.12 \\
.37 \\
.62 \\
.91 \\
1.25\end{array}$ & $\begin{array}{c}a m p / \mathrm{cm}^{2} \\
0.04\end{array}$ & Copper \\
\hline $\begin{array}{r}0.00 \\
3.83 \\
11.18 \\
21.05 \\
32.46 \\
44.12\end{array}$ & 0.025 & $\begin{array}{l}.025 \\
.018 \\
.008 \\
.001 \\
.000 \\
.000\end{array}$ & 0.0382 & $\begin{array}{r}0.00 \\
.28 \\
.68 \\
.96 \\
1.00 \\
1.00\end{array}$ & $\begin{array}{r}0.00 \\
.10 \\
.29 \\
.55 \\
.85 \\
1.15\end{array}$ & 0.01 & Nickel \\
\hline $\begin{array}{r}0.00 \\
3.83 \\
12.36 \\
21.05 \\
30.00 \\
40.00\end{array}$ & 0.040 & $\begin{array}{l}.040 \\
.030 \\
.018 \\
.009 \\
.002 \\
.000\end{array}$ & 0.0347 & $\begin{array}{r}0.00 \\
.25 \\
.55 \\
.77 \\
.95 \\
1.00\end{array}$ & $\begin{array}{r}0.00 \\
.11 \\
.35 \\
.61 \\
.86 \\
1.15\end{array}$ & 0.02 & Nickel \\
\hline $\begin{array}{r}0.00 \\
3.83 \\
12.36 \\
21.05 \\
30.00 \\
40.00\end{array}$ & 0.059 & $\begin{array}{l}.059 \\
.044 \\
.021 \\
.006 \\
.002 \\
.000\end{array}$ & 0.0315 & $\begin{array}{r}0.00 \\
.25 \\
.64 \\
.90 \\
.97 \\
1.00\end{array}$ & $\begin{array}{r}0.00 \\
.12 \\
.39 \\
.65 \\
.95 \\
1.26\end{array}$ & 0.04 & Nickel \\
\hline
\end{tabular}

TABLE 3. Reductions in the diffusion layer densities of the Brenner tests 


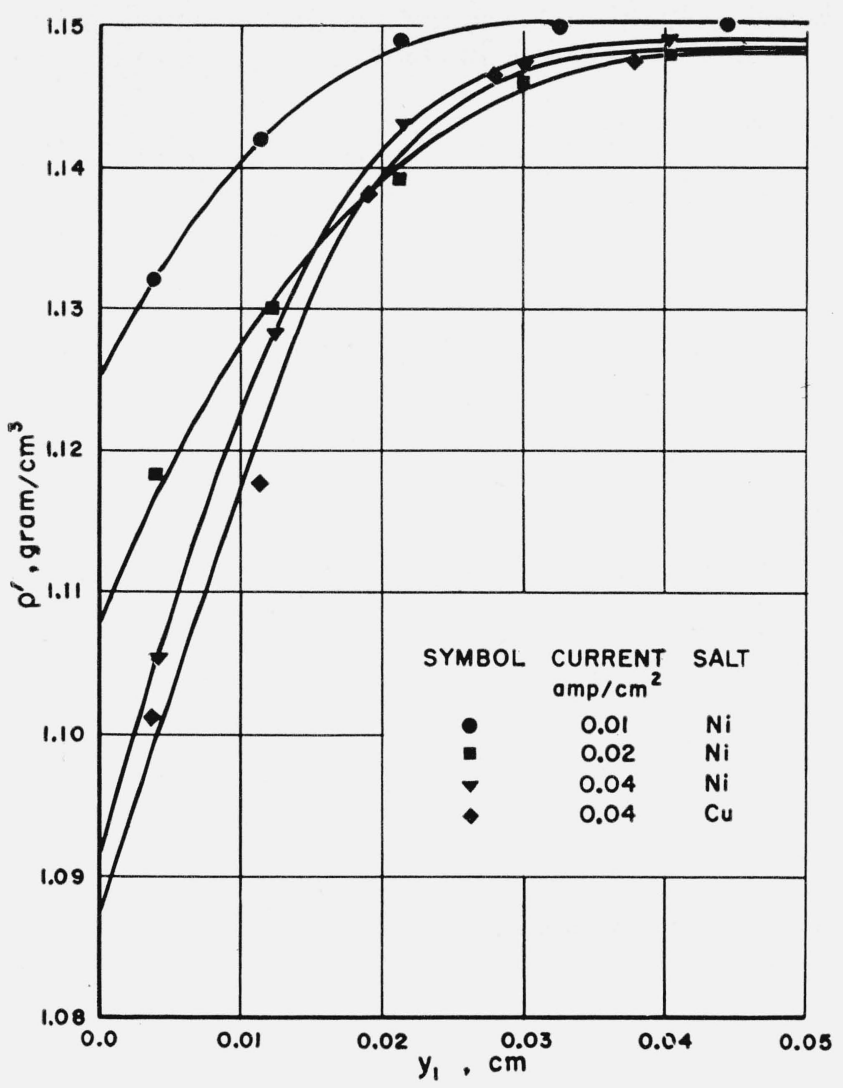

Figure 7. Graphical representation of the data from Brenner's tests.

in identifying the exact magnitude of the maximum layer thickness from the curves of figure 7 . If this element of uncertainty be ignored, the agreement between the theoretical and observed values may be judged to be fairly satisfactory.

The second question to be considered is the distribution of the mean densities in the layer itself, that is the variation of $\Delta \rho^{\prime} / \Delta \rho_{m}$ with $y_{1} / y_{s 1}$. The observed proportional density reductions for a given relative distance are given in table 3 . The method of determinations is obvious from the contents of the various columns. In forming the ratio $y_{1} / y_{s 1}$, the theoretical values of $y_{s 1}$ are used. Values from the table are plotted in figure 8. The curve drawn is the theoretical distribution and is the plot of eq 117. Again the agreement between observation and theory may be judged to be fairly satisfactory.

The third question to be examined is the depend-

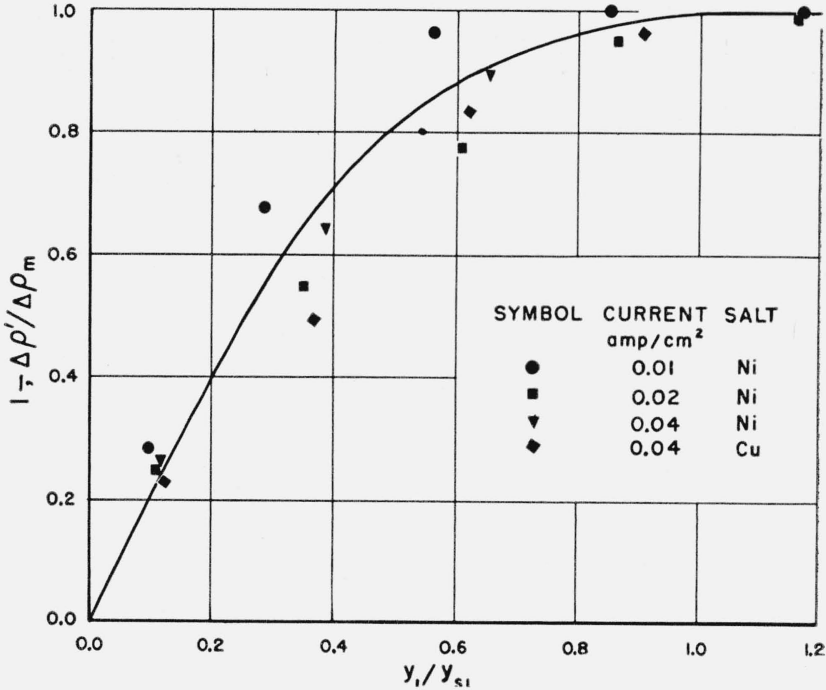

FIGURE 8. Distribution of densities of the diffusion layer according to theory and observation.

ence of the average density gradient at the cathode surface upon the intensity of the electrical current. This question deals with the electrochemical boundary condition represented by eq 37 . Since the average gradient according to eq 95 is

$$
\overline{\left(\frac{\partial \alpha}{\partial y}\right)_{0}}=-\frac{2.1}{y_{s l}}
$$

eq 37 may be changed to

$$
\frac{2.1 m D \Delta \rho_{m}}{y_{\mathrm{s} 1}}=\frac{U_{2}}{U_{1}+U_{2}} \frac{I}{F} .
$$

This relation may be examined in two ways. If the transport number of the cations (nickel and copper) are known, the relation may be used in computing $\Delta \rho_{m}$, the average density reduction at the cathode. Or, using the observed value $\Delta \rho_{m}$, the transport number of the cations may be determined. The second method will be used here.

From the data in table 3 the ratios $\Delta \rho_{m} / y_{s 1}$ are computed and these are plotted against current density in figure 9. The distribution of the points is linear, and the equation of the line drawn is

$$
\Delta \rho_{m} /\left(y_{s 1} I\right)=0.5 \times 10^{2} .
$$

\begin{tabular}{|c|c|c|c|c|c|c|c|}
\hline $\begin{array}{c}\text { Bath } \\
\text { density, } \\
\rho_{1}\end{array}$ & $\begin{array}{c}\text { Cathode } \\
\text { density, } \\
\rho_{0}\end{array}$ & $\begin{array}{c}\text { Reduction of } \\
\text { density at } \\
\text { cathode, } \\
\Delta \rho_{\mathrm{m}}\end{array}$ & $\begin{array}{c}\text { Cathode } \\
\text { immersion } \\
\text { length, } \\
l_{1}\end{array}$ & $\begin{array}{c}\text { Observed } \\
\text { maximum layer } \\
\text { thickness, } \\
y_{\diamond 1}\end{array}$ & $\begin{array}{c}\text { Theoretical } \\
\text { maximum layer } \\
\text { thickness, } \\
y_{s 1}\end{array}$ & $\begin{array}{c}\text { Current } \\
\text { intensity, } \\
I\end{array}$ & $\begin{array}{c}\text { Metallic } \\
\text { ion }\end{array}$ \\
\hline $\begin{array}{l}\mathrm{g} / \mathrm{cm}{ }^{3} \\
1.148 \\
1.150 \\
1.148 \\
1.149\end{array}$ & $\begin{array}{l}\mathrm{g} / \mathrm{cm}^{3} \\
1.087 \\
1.125 \\
1.108 \\
1.090\end{array}$ & $\begin{array}{l}\mathrm{g} / \mathrm{cm}{ }^{3} \\
0.061 \\
.025 \\
.040 \\
.059\end{array}$ & $\begin{array}{r}c m \\
10 \\
10 \\
10 \\
10\end{array}$ & $\begin{array}{c}c m \\
0.032 \\
.032 \\
.037 \\
.034\end{array}$ & $\begin{array}{c}c m \\
0.0302 \\
.0382 \\
.0347 \\
.0315\end{array}$ & $\begin{array}{c}a m p / c m{ }^{2} \\
0.04 \\
.01 \\
.02 \\
.04\end{array}$ & $\begin{array}{c}\text { Copper } \\
\text { Nickel } \\
\text { Do. } \\
\text { Do. }\end{array}$ \\
\hline
\end{tabular}

TABLE 4.-Theoretical and observed maximum diffusion layer thickness in the Brenner experiments 
We also have

and

$$
F \cdot D=2.56 \times 10^{-1}
$$

$$
m=1.32 \times 10^{-2} .
$$

Substituting these values in eq 118 , it is seen that

$$
\frac{U_{2}}{U_{1}+U_{2}}=0.356
$$

Accordingly, the transport of the cations, nickel, is $n_{c}=0.644$, a value very close to the generally accepted value determined by other methods. 'Thus in this respect also there is agreement between theory and observation.

Another element of the theory that could be examined with considerable signficance is the question of the vertical velocities. Unfortunately in the experiments of Brenner this matter was not touched. To give an idea of the order of magnitude of the velocities involved, we may compute the maximum upward velocity in a normal section of the diffusion layer using eq 99 . Consider the case of copper sulfate. Corresponding to the current intensity of $\mathrm{I}=4 \times 10^{-2} \mathrm{am}, \mathrm{p} / \mathrm{cm}^{2}$, the density reduction at the cathode surface is $\Delta \rho_{m} / \rho_{1}=5.31 \times 10^{-2}$. Since $1 / \gamma=$ $1.74 \times 10^{-4}$, the formula gives for maximum velocities

$$
\frac{u_{m}}{\sqrt{g x}}=2.50 \times 10^{-3} .
$$

Thus at a point $x=10 \mathrm{~cm}, u_{m}$ equals $2.48 \mathrm{~mm} / \mathrm{sec}$.

The discussion presented above must lead one to the following conclusion. The approximate analytical theory of the diffusion layer based on the assumption of a uniform density reduction along the entire cathode surface gives fairly satisfactory results regarding the maximum width of the layer, the average densities in planes parallel to the cathode surface, and the transport number of ions. Brenner is now engaged in further work on the variation of densities in planes parallel to the cathode surface. Information of this kind may possibly point to defects in the present theory. In that event it might be necessary to base the theoretical investigation of the problem on the assumption that the gradient of the density and not the density is uniform. along the cathode surface.

The author expresses his sincere appreciation to $\mathrm{W}$. Blum for the guidance shown in the beginning of the investigation, and to $\mathrm{A}$. Brenner for advice given

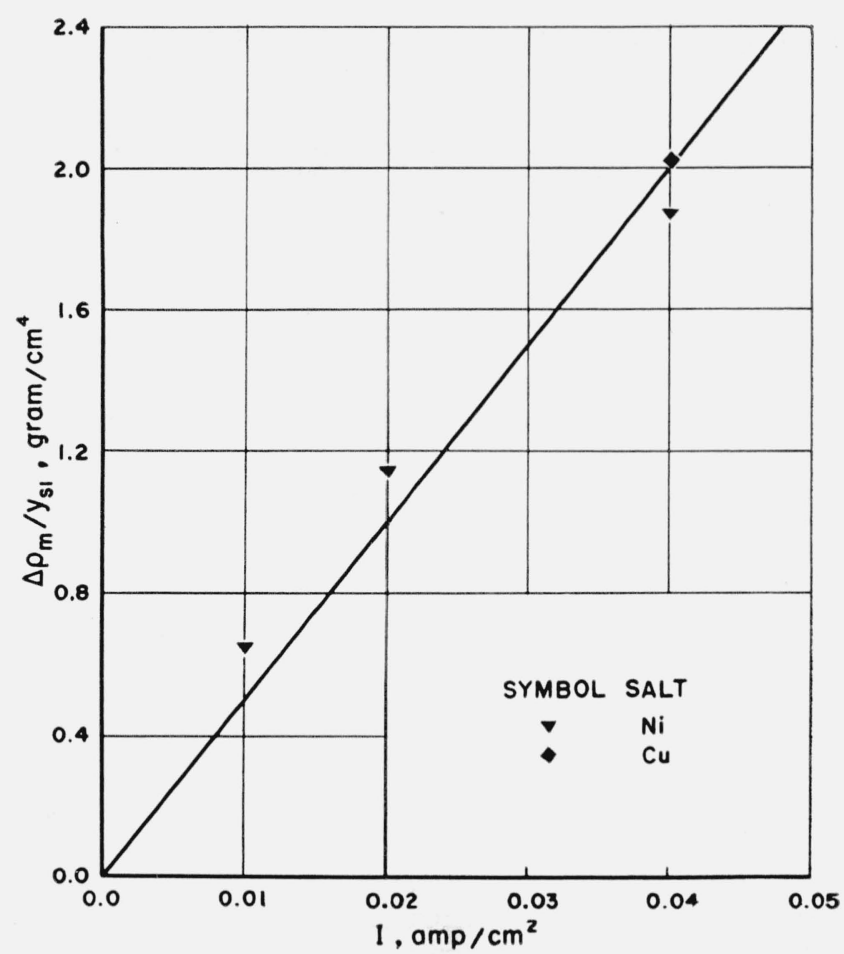

Figure 9. Relation of maximum density reduction to the intensity of electric current.

during the progress of work. Thanks are due also to K. Hilding Beij, and to John L. French for reviewing the paper.

\section{References}

[1] J. N. Agar, Diffusion and convection at electrodes, Discussions of the Faraday Society, No. 1 (1947), Electrode Processes, p 26.

[2] Carl Wagner, The role of natural convection in electrolytic processes, J. Electrochem. Soc. 95, 161 (1949).

[3] J. Larmor, Aether and matter, p. 289 (Cambridge University Press (Eng.), 1900).

[4] W. Nernst, Zur Kinetik der in Lösung befindlicher Körper, Z. Phys. Chem. 2 613 (1888).

[5] S. (ioldstein, Modern developments in fluid dynamies, II, 638 (Oxford, The Clarendon Press, 1938).

[6] Abner Brenner, Cathode films in electrodeposition, Proc. Educational Sessions of the Twenty-ninth Annual Convention, American Electroplaters Society, p. 28 (1941).

[7] Svante Arrhenius Úber die innere Reibung verdünnter wässeriger Lösungen, Z. Phys. Chem. 1285 (1887).

[8] Julius Wagner, Über die innere Reibung verdünnter Saltzlösungen, Z. Phys. Chem. 5 31 (1890).

[9] G. Thovert, Recherches sur la diffusion, Ann. Chem. Phys. [7] 26, 366 (1902).

Washington, January 17, 1951. 